\title{
TRANSLATION-INVARIANT FUNCTIONALS ON FUNCTIONS DEFINED IN EUCLIDEAN SPACES $\left({ }^{1}\right)$
}

BY

JOEL ALAN SMOLLER

1. Introduction. Let $S$ be a linear space of complex-valued functions defined on Euclidean $N$-space $R^{N}, N \geqq 1$. A subspace $X \neq 0$ of $S$ is called translation invariant (or simply invariant) if $f\left(x_{1}, \cdots, x_{N}\right) \in X$ implies

$$
f_{s_{1}, \cdots, s_{N}}\left(x_{1}, \cdots, x_{N}\right)=f\left(x_{1}-s_{1}, \cdots, x_{N}-s_{N}\right) \in X
$$

for all $\left(s_{1}, \cdots, s_{N}\right) \in R^{N}$. A linear functional $F$ on $X$ is called translationinvariant (or simply invariant) on $X$ if $F\left(f_{s_{1}, \cdots, s_{N}}\right)=F(f)$ for all $f \in X$ and all $\left(s_{1}, \cdots, s_{N}\right) \in R^{N}$. In the case where $X$ is an invariant subspace of $C_{c}\left(R^{1}\right)=$ continuous functions with compact support on $R^{1}$, Jerison and Rudin [2] have characterized all the invariant functionals on $X$ which arise from a measure on $R^{1}$. They have shown that the space of such functionals on $X$ is one-dimensional, and that if $\mu$ is a measure on $R^{1}$ which gives rise to an invariant functional on $X$, then there exists an integer $p \geqq 0$, depending only on $X$, and a scalar $\lambda$ depending on $\mu$ such that

$$
\mu(f)=\lambda \int_{-\infty}^{\infty} f(x) x^{p} d x, \quad f \in X .
$$

We shall extend the above result in many ways. First, instead of measures, we shall take Schwartz distributions $T$ which have Fourier transforms [3]. Then we shall also extend the class $X$ of functions from invariant subspaces of $C_{c}\left(R^{1}\right)$ to invariant subspaces of $L^{\#}\left(R^{1}\right)$, i.e., the space of functions $f$ on $R^{1}$ satisfying $x^{n} f(x) \in L_{1}, n=0,1, \ldots$. We shall also investigate invariant subspaces of $L^{\#}\left(R^{2}\right)$; i.e., the space of functions $f$ on $R^{2}$ satisfying $x^{n} y^{m} f(x, y)$ $\in L_{1}, n, m=0,1,2, \cdots$. However, in this case, we must at the outset give up any hope of obtaining a result stating that the invariant functionals always form a one-dimensional space as the following example showsi Let $g$ and $h$ be the functions defined by

$$
\begin{aligned}
g(x, y) & =\left(x^{2}-1\right) \sin y, & & -1 \leqq x \leqq 1,-\pi \leqq y \leqq \pi, \\
& =0, & & \text { elsewhere, } \\
h(x, y) & =\left(y^{2}-1\right) \sin x, & & -1 \leqq y \leqq 1,-\pi \leqq x \leqq \pi, \\
& =0, & & \text { elsewhere. }
\end{aligned}
$$

Presented to the Society, June 25, 1962, under the title Translation-invariant linear functionals on spaces of functions of two variables; received by the editors July 22, 1963.

( ${ }^{1}$ This work was supported by the National Science Foundation grant number G-7430 and constitutes the major portion of the author's doctoral dissertation at Purdue University. The author wishes to thank Professor Meyer Jerison for his guidance and understanding. 
Let $X$ be the invariant subspace of $L^{\#}\left(R^{2}\right)$ satisfying $\hat{f}(0,0)=0$ for all $f$ in $X$, where $\hat{f}$ denotes the Fourier transform of $f$. Then $g$ and $h$ are in $X$. Define functionals $F_{1,0}$ and $F_{0,1}$ on $X$ by

$$
\begin{array}{ll}
F_{0,1}(f)=(-i)[\partial \hat{f}(x, y) / \partial y]_{x=0, y=0}, & f \in X, \\
F_{1,0}(f)=(-i)[\partial \hat{f}(x, y) / \partial x]_{x=0, y=0}, & f \in X .
\end{array}
$$

Then it is easy to see that these functionals are invariant on $X$. Moreover, $F_{1,0}(g)=F_{0,1}(h)=0$ and $F_{1,0}(h) \neq 0, F_{0,1}(g) \neq 0$, so $F_{0,1}$ and $F_{1,0}$ are linearly independent on $X$. Therefore the space of invariant functionals on $X$ is at least two-dimensional.

What we shall show, however, is that if $X$ is an invariant subspace of $L^{\#}\left(R^{2}\right)$ and if $(0,0)$ is an isolated point of

$$
\bigcap_{f \in X}\left\{(s, t) \in R^{2}: \hat{f}(s, t)=0\right\}
$$

then any invariant functional $F$ on $X$ which comes from the class of Schwartz distributions which have Fourier transforms satisfies the following: there exists a differential operator $D$ such that

$$
T(f)=(D \hat{f})(0,0), \quad f \in X .
$$

Moreover, the space of such invariant functionals will be shown to have finite dimension and we shall obtain the best upper bound for the dimension.

It is assumed in this paper that the reader has a knowledge of the basic properties of Fourier transforms as in [1] and of the theory of distributions as in [3]. In particular, we shall use the notations in [3] for the various spaces of distributions.

2. Distributions applied to the study of invariant functionals. In this section we shall give a proof of a theorem similar to Theorem 4 in [2]. The importance of this result is that the method of proof generalizes to functionals on invariant subspaces of functions defined on $R^{N}, N>1$.

Definition 2.1. $L^{\#}\left(R^{N}\right)$ is a class of functions defined on $R^{N}$. A function $f \in L^{\#}\left(R^{N}\right)$ if for every ordered set of non-negative integers $p=\left(p_{1}, \cdots, p_{N}\right)$, we have $x_{1}^{p_{1}} \ldots x_{N}^{p_{N}} f\left(x_{1}, \cdots, x_{N}\right) \equiv x^{p} f(x) \in L_{1}\left(R^{N}\right)$, where $L_{1}\left(R^{N}\right)$ is the space of Lebesgue integrable functions on $R^{N}$.

When there is no chance of confusion we shall write $L^{\#}=L^{\#}\left(R^{N}\right)$. We note that $C_{c} \subset L^{\#}$, where $C_{c}=C_{c}\left(R^{N}\right)$ denotes the space of continuous functions with compact support on $R^{N}$. Our invariant subspaces $X$ will always be subspaces of $L^{\#}$. The main property that we want to prove about $L^{\#}$ is that if $f \in L^{\#}, T \in\left(\mathscr{S}^{\prime}\right)(=$ space of Schwartz distributions having Fourier transforms), then the "exchange rule" holds, i.e.,

$$
(f * T)^{\wedge}=\hat{f} \hat{T}
$$


where "*" denotes the convolution product in the sense of distributions and the product on the right is distribution multiplication.

Lemma 2.1. If $f \in L^{\#}$ and $T \in\left(\mathscr{S}^{\prime}\right)$, then $(f * T) \in\left(\mathscr{S}^{\prime}\right)$ and $(f * T)^{-}$ $=\hat{f} \hat{T}$.

Proof. It is enough to show that $f$ is rapidly decreasing, i.e., $f \in\left(\mathscr{O}_{c}^{\prime}\right)$ $\left[3\right.$, Tome II ]. Thus let $k$ be a non-negative integer and put $g(x)=\left(1+x^{2}\right)^{k^{k} / 2} \hat{f}(x)$; we are to show $g \cdot \phi_{n} \rightarrow 0$ if $\phi_{n}$ is a sequence of functions converging to zero in $\left(\mathscr{D}_{L_{1}}\right)$. But this is clear since $\phi_{n}$ converges to zero in $\left(\mathscr{D}_{L_{\infty}}\right)$ and $g \in L_{1}$.

The next result connects the notions of invariance and convolution product. We first define $f^{-}(x)=f(-x)$.

Theorem 2.2. Let $T \in\left(\mathscr{S}^{\prime}\right)$ and let $X$ be an invariant subspace of $L^{\#}$. Then for each $f \in X$ there exists a constant $c_{f}$ such that $T * f^{-}=c_{f}$ if and only if $T$ can be extended to an invariant functional on $X$.

Proof. Suppose $T$ can be extended to an invariant functional on $X$. Then if $\phi \in(\mathscr{D})$,

$$
\begin{aligned}
\left(T * f^{-}\right) \cdot \phi & =T_{\xi} \cdot \int f^{-}(\eta) \phi(\xi+\eta) d \eta=T_{\xi} \cdot \int f(-\eta) \phi(\xi+\eta) d \eta \\
& =T_{\xi} \cdot \int f(\xi-\eta) \phi^{-}(-\eta) d \eta=\left(T * \phi^{-}\right) \cdot f \\
& =\left(\phi^{-} * T\right) f=\phi_{\eta}^{-} \cdot\left[T_{\xi} \cdot f(\xi+\eta)\right] \\
& =\phi^{-} \cdot c_{f}=c_{f} \cdot \phi .
\end{aligned}
$$

Therefore, $T * f^{-}=c_{f}$.

Conversely, suppose $T * f^{-}=c_{f}$ for all $f \in X$. We define $T$ on $X$ by $T(f)$ $=c_{f}, f \in X$. (We note that this definition is consistant if $f \in(\mathscr{S})$ for if $f \in(\mathscr{S})$ and $\phi \in(\mathscr{D})$, then as above, $c_{f} \cdot \phi=\left(T * f^{-}\right) \cdot \phi=\left(T * \phi^{-}\right) \cdot f$. Now let $\phi \rightarrow \delta$ in $\left(\mathscr{D}^{\prime}\right)$ where $\delta$ is the Dirac measure, and $\mathcal{S} \phi=1$. Then since $\phi^{-} \rightarrow \delta$ and $T * \delta=T$ we get $c_{f}=(T * \delta) \cdot f=T \cdot f$.) We shall now show $T\left(f_{t}\right)=T(f)$ for all $t$ and all $f \in X$. We define $f_{t}^{-}(y)=f(-t-y)$ for all $y$ and $t$. Then if $\phi \in(\mathscr{D})$,

$$
\begin{aligned}
c_{f_{t}} \cdot \phi & =\left(T * f_{t}^{-}\right) \cdot \phi=T_{x} \cdot \int f_{t}^{-}(y) \phi(x+y) d y \\
& =T_{x} \cdot \int f(-t-y) \phi(x+y) d y \\
& =T_{x} \cdot \int f(z) \phi_{t}(x-z) d z=T_{x} \cdot \int f^{-}(-z) \phi_{t}(x-z) d z \\
& =\left(T * f^{-}\right) \cdot \phi_{t}=c_{f} \cdot \phi_{t}=c_{f} \cdot \phi .
\end{aligned}
$$

Therefore $c_{f_{t}}=c_{f}$, i.e., $T(f)=T\left(f_{t}\right)$ so $T$ is invariant on $X$. 
Therefore if $T \in\left(\mathscr{S}^{\prime}\right)$ is an invariant functional on an invariant subspace $X \subset L^{\#}$ then the invariance condition can be expressed by $f^{-} * T$ $=c_{f}, f \in X$.

Theorem 2.3. Suppose $X$ is an invariant subspace of $L^{\#}$ and $T \in\left(\mathscr{S}^{\prime}\right)$ is an invariant functional on $X$. If $g(0) \neq 0$ for some $g \in X$, then there is a constant $\lambda$ such that

$$
T(f)=\lambda \int f(x) d x, \quad f \in X .
$$

Proof. We have $f^{-} * T=c_{f}$ for all $f \in X$. If we take the Fourier transform of both sides and use the exchange rule, we get $\hat{f}^{-} \hat{T}=c_{f} \delta$. Multiplying both sides of this equation by $\hat{g}^{-}$gives $\hat{g}^{-} \hat{f}^{-} \hat{T}=c_{f} \hat{g}^{-} \delta$, and by symmetry, $\hat{f}^{-} \hat{g}^{-} \hat{T}=c_{g} f^{-} \delta$. Therefore $c_{f} \hat{g}^{-} \delta=c_{g} \hat{f}^{-} \delta$ so that $c_{f} \hat{g}^{-}(0)=c_{g} \hat{f}^{-}(0)$. But then $c_{f} \hat{g}(0)=c_{g} \hat{f}(0)$ and this implies (2) if $\hat{g}(0) \neq 0$.

This theorem shows that there is no interest in studying invariant functionals on invariant subspaces $X$ of $L^{\#}$ in which $g(0) \neq 0$ for some $g \in X$. Therefore we shall always assume that $\hat{f}(0)=0$ for all $f \in X$.

Our next objective is to prove an analogue of Theorem 4 in [2] for invariant functionals $T \in\left(\mathscr{S}^{\prime}\right)$ on invariant subspaces of $L^{\#}\left(R^{1}\right)$. For each $f \in X$, we define $Z_{f}=\left\{t \in R^{1}: f(t)=0\right\}$, and we put $Z=\bigcap_{f \in X} Z_{f}$.

Theorem 2.4. Let $X$ be an invariant subspace of $L^{\#}\left(R^{1}\right)$ and let $T \in\left(\mathcal{S}^{\prime}\right)$ be invariant on $X$. If $D^{i} f(0)=0$ for all $f \in X$ and all $i \geqq 0$, then $T(f)=0$ for all $f \in X$. If, on the other hand, there is a smallest integer $p>0$ such that $D^{p} \hat{g}(0) \neq 0$ for some $g \in X$, then there exists a constant $\lambda$ such that

$$
T(f)=\lambda \int_{-\infty}^{\infty} x^{p} f(x) d x, \quad f \in X .
$$

(We remark that if there is a $g \not \equiv 0$ with $g \in X \cap C_{c}\left(R^{1}\right)$, then $g$ is extendable to an entire analytic function so for this $g$ we cannot have $D^{i} \hat{g}(0)=0$ for all $i=0$ and therefore (3) holds. This is the same conclusion as Theorem 4 of [2].)

Proof. We have $f^{-} * T=c_{f}$ for each $f \in X$. We take the Fourier transform of both sides of this equation and apply the exchange rule to get

$$
\hat{f}^{-} \hat{T}=c_{f} \delta \quad(f \in X),
$$

where $\delta$ is the Dirac measure. We divide the proof into two cases: 0 is a limit point in $Z$ or 0 is an isolated point in $Z$.

Suppose first that 0 is a limit point in $Z$. Then there exist $y_{n} \in Z$ such that $y_{n} \rightarrow 0$ and $f^{-}\left(y_{n}\right)=0, n=1,2, \cdots$, for each $f \in X$. Therefore $D^{1} \hat{f}(0)$ $=0$ and by the theorem of the mean, $D^{i} \hat{f}(0)=0$ for all $i \geqq 0$. Thus we shall show $c_{f}=0$.

Choose $\psi \in C^{\infty}, \psi(0)=1$, support of $\psi \subset[-1,1]$. Then $\left\|D^{k} \psi\right\|_{\infty} \leqq A_{k}$, 
$k=0,1, \ldots$. Put $\phi_{n}(x)=\psi(n x), n=1,2, \ldots$. Then the support of $\phi_{n}$ is in $[-1 / n, 1 / n], D^{k} \phi_{n}(x)=n^{k} D^{k} \psi(n x)$ so $\left\|D^{k} \phi_{n}\right\|_{\infty} \leqq n^{k} A_{k}, k=0,1, \ldots$. Now from (4) we have $c_{f}=c_{f} \psi(0)=c_{f} \phi_{n}(0)=\hat{T} \hat{f}^{-} \phi_{n}$, so in order to prove that $c_{f}=0$, it suffices to show $\hat{f}^{-} \phi_{n} \rightarrow 0$ in $(\mathscr{D})$, i.e., $\left\|D^{p}\left(\hat{f}^{-} \phi_{n}\right)\right\|_{\infty} \rightarrow 0$ for $p=0,1, \cdots$. Write $\hat{f}^{-}(x)=x^{p+1} g(x)$ where $g \in C^{\infty}$, and $\left\|D^{q} g\right\|_{\infty} \leqq B_{q}, q=0,1,2, \ldots$. Then if $x \in[-1 / n, 1 / n]$ we can make an estimate of the form $\left|D^{p}\left(\hat{f}^{-} \phi_{n}\right)(x)\right|$ $\leqq c / n$, where $c$ is independent of $x$; thus $\left\|D^{p}\left(\hat{f}^{-} \phi_{n}\right)\right\|_{\infty} \rightarrow 0$.

Now suppose that 0 is isolated in $Z$. We shall show that the support of $\hat{T}$ is contained in $\bigcap_{f \in X} Z_{f}^{-}$. If $x_{0} \notin Z_{f}^{-}$, then there is a neighborhood $V$ of $x_{0}$ disjoint from $Z_{f}^{-}$. Let $\phi \in\left(\mathscr{D}_{V}\right)$; we shall show $\hat{T} \cdot \phi=0$. We define $\alpha(x)=\phi(x)\left[\hat{f}^{-}(x)\right]^{-1}$ if $x \in V$ and $\alpha(x)=0$ if $x \notin V$. Then $\alpha \in\left(\mathscr{D}_{V}\right), \alpha \hat{f}^{-}=\phi$ and since $0 \notin V, 0=c_{f} \alpha(0)=\hat{f}^{-} \hat{T} \alpha=\hat{T} \cdot \phi$. Hence $x_{0} \notin$ support of $\hat{T}$ so we have shown that the support of $\hat{T}$ is in $Z_{f^{-}}$, and consequently the support of $T$ is contained in $-Z$. Since 0 is isolated in $Z, 0$ is isolated in $-Z$ so we can find a neighborhood $U$ of 0 such that the support of $T$ meets $U$ only at 0 . Hence in $U$ we have $[1$, p. 100]

$$
T=\sum_{n=0}^{r} a_{n} D^{n} \delta, \quad a_{n} \text { constants. }
$$

Now if $\phi \in\left(\mathscr{D}_{U}\right)$, then since $D^{j} \hat{f}^{-}(0)=(-1)^{j} D^{j} \hat{f}(0)$, (4) and (5) give

$$
c_{f} \phi(0)=\sum_{n=0}^{r} a_{n}(-1)^{n} \sum_{j=0}^{n}\left(\begin{array}{l}
n \\
j
\end{array}\right)(-1)^{j}\left(D^{j} \hat{f}(0)\right)\left(D^{n-j} \phi(0)\right) \text {. }
$$

Now if $D^{i} f(0)=0$ for all $f \in X$ and all $i \geqq 0$, then (6) yields $c_{f}=0$ for all $f \in X$ so $T(f)=0$ for all $f \in X$. If on the other hand there is a smallest integer $p \geqq 0$ such that $D^{p} \hat{g}(0) \neq 0$ for some $g \in X$, then if $r<p$ the theorem holds with $\lambda=0$. If $r>p$, then if $\phi \in\left(\mathscr{D}_{U}\right)$ with $D^{i} \phi(0)=0$ if $0 \leqq i$ $<r-p$ and $D^{r-p} \phi(0) \neq 0$, then for this $\phi$ with $f=g$ in (6) we get $a_{r}=0$. Similarly $a_{r-1}=\cdots=a_{p+1}=0$, so that in (6) we may assume $r=p$, and hence (6) becomes $c_{f} \phi(0)=a_{p}\left[D^{p} \hat{f}(0)\right]_{\phi}(0)$. If we put $f=g$ in this equation, we get $a_{p}=c_{g}\left[D^{p} g(0)\right]^{-1}$ so that $c_{f}=c_{g}\left[D^{p} g(0)\right]^{-1} D^{p} \hat{f}(0)$ and this is (3).

In the case where $X \subset L^{\#}\left(R^{2}\right)$ our example in the introduction shows that the situation is quite different. We do have, however, the following theorem.

Theorem 2.5. Let $X$ be an invariant subspace of $L^{\#}\left(R^{2}\right)$ such that $(0,0)$ is an isolated point of $\bigcap_{f \in X} Z_{f}=\bigcap_{f \in X}\left\{(s, t) \in R^{2}: \hat{f}(s, t)=0\right\}$, and let $T$ $\in\left(\mathscr{S}^{\prime}\right)$ be invariant on $X$. Then there is an integer $t \geqq 0$ and constants $a_{i, j}$ such that

$$
T(f)=\sum_{i+j \leqq t} a_{i, j} D^{(i, j)} \hat{f}(0,0), \quad f \in X
$$

Proof. As in the proof of the last theorem, we have 


$$
\hat{f}^{-} \hat{T}=c_{f} \delta \quad(f \in X)
$$

and this implies that in a neighborhood $U$ of $(0,0)$, we have

$$
T=\sum_{i+j \leqq t} a_{i, j} D^{(i, j)} \delta .
$$

We choose $\phi \in\left(\mathscr{D}_{U}\right)$ such that $\phi(0,0) \neq 0$ and $D^{(i, j)} \phi(0,0)=0$ if $0<i+j$ $\leqq t$. Then if $f \in X$,

$$
c_{f} \phi(0,0)=c_{f} \delta \cdot \phi=\hat{f}^{-} \hat{T} \cdot \phi=\hat{T} \cdot \hat{f}^{-} \phi .
$$

Then from (9) we get

$$
\begin{aligned}
\hat{T} \cdot \hat{f}^{-} \phi & =\sum_{i+j \leqq t} a_{i, j} D^{(i, j)} \delta\left(f^{-} \phi\right)=\sum_{i+j \leqq t} a_{i, j}(-1)^{i+j} \delta \cdot\left[D^{(i, j)}\left(\hat{f}^{-} \phi\right)\right] \\
& =\sum_{i+j \leqq t} a_{i, j}(-1)^{i+j}\left[\sum_{a=0}^{j} \sum_{b=0}^{i}\left(\begin{array}{l}
j \\
a
\end{array}\right)\left(\begin{array}{l}
i \\
b
\end{array}\right)\left(D^{(b, a)} \hat{f}^{-}(0,0)\right)\left(D^{(i-b, j-a)} \phi(0,0)\right)\right] .
\end{aligned}
$$

Now if $0<(i-b)+(j-a) \leqq t$, then $D^{(i-b, j-a)} \phi(0,0)=0$; hence

$$
c_{f} \phi(0,0)=\sum_{i+j \leqq t} a_{i, j}(-1)^{i+j}\left(D^{(i, j)} \hat{f}^{-}(0,0)\right) \phi(0,0) .
$$

But $D^{(i, j)} \hat{f}^{-}(0,0)=(-1)^{i+j} D^{(i, j)} \hat{f}(0,0)$ so

$$
c_{f}=\sum_{i+j \leqq t} a_{i, j} D^{(i, j)} \hat{f}(0,0),
$$

which gives the desired result.

Under the hypotheses of this theorem, we see that $T(f)$ is given by a finite linear combination of derivatives of Fourier transforms of $f$ evaluated at the origin. Our task in the remainder of this paper is to show that these functionals form a finite-dimensional space and to find the best upper bound for the dimension of this space.

3. A class of invariant functionals. In this section we shall investigate the class of invariant functionals on an invariant subspace $X$, generated by functionals of the form

$$
\begin{aligned}
F_{p, q}(f) & =(i)^{-(p+q)}\left[\partial^{p+q} \hat{f}(x, y) / \partial x^{p} \partial y^{q}\right]_{x=0, y=0} \\
& =\int_{-\infty}^{\infty} \int_{-\infty}^{\infty} x^{p} y^{q} f(x, y) d x d y,
\end{aligned}
$$

where $p$ and $q$ are non-negative integers, $f \in X$ and $\hat{f}$ denotes the Fourier transform of $f$. The main result of this section is to obtain a necessary and sufficient condition for a finite linear combination of functionals of the type (11) to be translation-invariant on $X$.

From now on $X$ will denote a fixed invariant subspace of $L^{\#}\left(R^{2}\right) ; I, R$ 
and $C$ will denote the non-negative integers, the reals and the complex numbers, respectively. For every pair $(p, q) \in I^{2}$ we have the linear functionals $F_{p, q}$ on $X$ defined by (11). In view of Theorem 2.3 we see that there is nothing of interest in studying those $X$ for which $F_{0,0} \not \equiv 0$ on $X$ so we shall assume that $F_{0,0} \equiv 0$ on $X$. Furthermore, we may assume that $F_{p, q} \not \equiv 0$ on $X$ for some $(p, q) \in I^{2}$. Then we have integers $m, n, k$ in $I$ with the properties that

$$
\begin{aligned}
& m+n=k>0, F_{m, n} \not \equiv 0 \text { on } X \\
& F_{i, j} \equiv 0 \text { on } X \text { if }(i, j) \in I^{2}, i+j<k .
\end{aligned}
$$

For $(u, v) \in R^{2}$, we define the translation operators $T(u, v)$ on the set of functionals $F_{p, q}$ by

$$
\left[T(u, v) F_{p, q}\right](f)=F_{p, q}\left(f_{u, v}\right),
$$

where $f \in X$ and $f_{u, v}(x, y)=f(x-u, y-v)$. Expanding the right side of (13) and using (11) gives

$$
\begin{aligned}
T(u, v) F_{p, q}(f) & =\int_{-\infty}^{\infty} \int_{-\infty}^{\infty} x^{p} y^{q} f(x-u, y-v) d x d y \\
& =\int_{-\infty}^{\infty} \int_{-\infty}^{\infty}(x+u)^{p}(y+v)^{q} f(x, y) d x d y \\
& =\sum_{a=0}^{p} \sum_{b=0}^{q}\left(\begin{array}{l}
p \\
a
\end{array}\right)\left(\begin{array}{l}
q \\
b
\end{array}\right) F_{a, b}(f) u^{p-a} v^{q-b}
\end{aligned}
$$

We then have the basic

Lemma 3.1. The functional $F_{p, q}$ is invariant on $X$ if and only if $F_{a, b} \equiv 0$ on $X$ for every $(a, b) \in I^{2}$ with $a+b<p+q, a \leqq p, b \leqq q$.

Proof. $F_{p, q}$ is invariant on $X$ if and only if for all $(u, v) \in R^{2}$ and all $f \in X$, we have $F_{p, q}(f)=T(u, v) F_{p, q}(f)$. But from (14), this means that

$$
F_{p, q}(f)-\sum_{a=0}^{p} \sum_{b=0}^{q}\left(\begin{array}{l}
p \\
a
\end{array}\right)\left(\begin{array}{l}
q \\
b
\end{array}\right) F_{a, b}(f) u^{p-a} v^{q-b}=0
$$

for all $f \in X$ and all $(u, v) \in R^{2}$. Therefore $F_{p, q}$ is invariant on $X$ if and only if $F_{a, b}(f)=0$ for all $f \in X$ and all $(a, b) \in I^{2}, a+b<p+q, a \leqq p, b \leqq q$.

Corollary 3.2. Let $m$ and $n$ be the integers defined in (12). Then the functional $F_{m, n}$ is invariant on $X$.

Let $\mathscr{F}=\mathscr{F}(X)$ denote the nonzero invariant functionals on $X$ of the type $F_{p, q}$. The next two lemmas will show that $\mathscr{F}$ has at most $k+1$ elements.

Lemma 3.3. Let $F_{r, s}$ and $F_{p, q}$ be in $\mathscr{F}$. Then $p>r$ if and only if $q<s$. 
Proof. We may assume that $p+q \geqq r+s$. Suppose $p>r$. If $q \geqq s$ then $p+q>r+s$ so by Lemma 3.1, $F_{r, s} \equiv 0$, a contradiction; hence $q<s$. Conversely, if $q<s$, then $s+p>q+p \geqq r+s$ so $p>r$.

It follows from this lemma that $p<r$ if and only if $q>s$. It is this fact which enables us to prove the next lemma on the cardinality of $\mathscr{F}$. Recall that $k$ is defined in (12).

Lemma 3.4. There are at most $k+1$ elements in $\mathscr{F}$.

Proof. We know $F_{m, n} \in \mathscr{F}$ and if $F_{p, q} \in \mathscr{F}$, then $p+q \geqq k=m+n$. Thus if $(p, q) \neq(m, n)$, then either $p<m$ and $q>n$ or $p>m$ and $q<n$. Let

$$
\begin{array}{rlrl}
A_{i} & =\left\{F_{i, s}: F_{i, s} \text { is invariant on } X, s>n\right\}, & i=0,1, \cdots, m-1, \\
B_{j}=\left\{F_{t, j}: F_{t, j} \text { is invariant on } X, t>m\right\}, & j=0,1, \cdots, n-1 .
\end{array}
$$

(By agreement, if $m=0$ or $n=0$, then the $A_{i}$ 's or $B_{j}$ 's are empty, respectively.) By Lemma 3.3, every element of $\mathscr{F}$ distinct from $F_{m, n}$ (i.e., every $F_{p, q} \in \mathscr{F}$ with $\left.(p, q) \neq(m, n)\right)$ must be in an $A_{i}$ or in a $B_{j}$. From Lemma 3.1, for every fixed $i$ and $j$, the sets $\mathscr{F} \cap A_{i}$ and $\mathscr{F} \cap B_{j}$ can have at most one element. Hence there are at most $m+n+1=k+1$ elements in $\mathscr{F}$.

These two lemmas enable us to easily construct sets $\mathscr{F}$. For example, we let

$$
X=\left\{f \in L^{\#}\left(R^{2}\right): F_{0,0}(f)=F_{0,1}(f)=F_{1,0}(f)=F_{2,0}(f)=0\right\}
$$

and then $k=2, \mathscr{F}=\mathscr{F}(X)=\left\{F_{0,2}, F_{1,1}, F_{3,0}\right\}$. On the other hand, there is no space $X$ for which $\mathscr{F}(X)=\left\{F_{0,2}, F_{1,1}, F_{2,1}\right\}$, for by the previous lemmas $F_{1,1}$ and $F_{2,1}$ cannot both be nonzero and invariant. Finally, there are spaces $X$ for which $\mathscr{F}(X)$ has less than $k+1$ elements. For example, let

$$
X=\left\{f \in L^{\#}\left(R^{2}\right): F_{0, j}(f)=0 \text { for all } j \in I\right\} .
$$

Then since the function $g(x, y)$ defined by $g(x, y)=\sin x \sin y$ if $|x| \leqq \pi$, $|y| \leqq \pi / 2$ and zero elsewhere is in $X$ and $F_{1,0}(g) \neq 0$, we see that $k=1$, $\mathscr{F}(X)=\left\{F_{1,0}\right\}$ and $\mathscr{F}(X)$ has less than $k+1$ elements.

For later use we formally state the next obvious corollary. (Here the brackets denote the linear hull of a set of vectors, i.e., the space of finite linear combinations of elements of the set.)

Corollary 3.5. Let $\mathscr{K}=\mathscr{K}(X)=\left\{F_{i, k-i} \in \mathscr{F}\right\}$. Then $\operatorname{dim}[\mathscr{K}] \leqq k+1$.

This completes our investigation of the set $\mathscr{F}$. We now want to prove some generalizations of Lemma 3.1. This is necessary because we shall work with invariant functionals which are finite linear combinations of the $F_{p, q}$ 's. In what follows we assume that $k$ is the unique integer defined in (12).

Let $t$ be a positive integer, $t>k$ and let the functional $F$ on $X$ be defined by 


$$
F=\sum_{i=0}^{t} \theta_{i} F_{i, t-i}, \quad \theta_{i} \in C
$$

$F$ is invariant on $X$ if and only if, for all $(u, v) \in R^{2}, T(u, v) F=F$ on $X$. Since $T(u, v)$ is linear, we use (14) to conclude that if $F$ is invariant on $X$, then

$$
\sum_{i=0}^{t} \theta_{i} \sum_{a=0}^{i} \sum_{b=0}^{t-i}\left(\begin{array}{c}
i \\
a
\end{array}\right)\left(\begin{array}{c}
t-i \\
b
\end{array}\right) F_{a, b}(f) u^{i-a} v^{t-i-b}=\sum_{i=0}^{t} \theta_{i} F_{i, t-i}(f)
$$

for all $(u, v) \in R^{2}$ and all $f \in X$. By definition of $k, F_{a, b}(f)=0$ if $a+b<k$. Therefore in (16) we may assume that only those terms with $0<(i-a)$ $+(t-i-b) \leqq t-k$ appear. The coefficient of $u^{\lambda} v^{\nu}$ in (16) is

$$
\sum_{i=\lambda}^{t-\nu} \theta_{i}\left(\begin{array}{c}
i \\
i-\lambda
\end{array}\right)\left(\begin{array}{c}
t-i \\
t-\nu-i
\end{array}\right) F_{i-\lambda, t-\nu-i}(f),
$$

and since (16) holds for all $(u, v) \in R^{2}$, we see that this coefficient must vanish. Since this is true for all $f \in X$, we conclude

$$
\sum_{i=\lambda}^{t-\nu} \theta_{i}\left(\begin{array}{c}
i \\
i-\lambda
\end{array}\right)\left(\begin{array}{c}
t-i \\
\nu
\end{array}\right) F_{i-\lambda, t-\nu-i} \equiv 0 \text { on } X,
$$

for every pair $(\lambda, \nu) \in I^{2}, 0<\lambda+\nu \leqq t-k$. Conversely, if (16) holds for every $(\lambda, \nu) \in I^{2}, 0<\lambda+\nu \leqq t-k$, then it is easy to see that $F$ is invariant on $X$. Thus we have

Lemma 3.6. The functional $F=\sum_{i=0}^{t} \theta_{i} F_{i, t-i}, t>k, \theta_{i} \in C$ is invariant on $X$ if and only if for every pair $(\lambda, \nu) \in I^{2}$ with $0<\lambda+\nu \leqq t-k$, (17) holds.

Since we shall later be interested in functionals which are linear combinations of functionals of the form (15), we shall obtain a necessary and sufficient condition for the invariance of functionals of this type. We make the convention that $F_{i, j} \equiv 0$ if $i<0$ or $j<0$.

Let $t=t_{1}>t_{2}>\cdots>t_{p}>k$ be positive integers and let

$$
G_{j}=\sum_{i=0}^{t_{j}} \theta_{i}^{j} F_{i, t_{j}-i} \quad\left(\theta_{i}^{j} \in C\right), 1 \leqq j \leqq p .
$$

We define the functional $F$ on $X$ by

$$
F=\sum_{j=1}^{p} G_{j}
$$

We define the coefficients of $F$ on level $s, 0 \leqq s \leqq t$, to be those $\theta_{i}^{j}$ with $i+j=s$. We then have the following lemma. 
Lemma 3.7. The functional $F$ defined by (18) and (19) is invariant on $X$ if and only if for every $(\lambda, \nu) \in I^{2}$ with $0<\lambda+\nu<t-k$ we have

$$
0=\sum_{j=1}^{p} \sum_{i=\lambda}^{t_{j}-\nu} \theta_{i}^{j}\left(\begin{array}{c}
i \\
i-\lambda
\end{array}\right)\left(\begin{array}{c}
t_{j}-i \\
t_{j}-\nu-i
\end{array}\right) F_{i-\lambda, t_{j}-\nu-i} \quad(\text { on } X) .
$$

The proof is straightforward and we omit the details.

Now we can write any functional of the form (19) as

$$
F=\sum_{i=0}^{t} \theta_{i} F_{i, t-i}+F_{t-1}
$$

where $F_{t-1}=\sum_{j=2}^{p} G_{t j}, \theta_{i}=\theta_{i}^{1}, 0 \leqq i \leqq t$. We then make the following definition.

Definition 3.8. Let $F$ be a functional defined by (21). If $F$ has a nonzero coefficient on level $t$, we say that (16) is a representation of $F$ of order $t$, and we call $F-F_{t-1}$ the principal part of this representation.

We then have the following corollary to Lemma 3.7.

Corollary 3.9. Let $F$ be an invariant functional on $X$ having a representation of order $t>k$ given by (21). Then the coefficient of $u^{\lambda} v^{\nu}(0<\lambda+\nu \leqq t-k)$ in the equation $F\left(f_{u, v}\right)=F(f)$ is

$$
0=\left\{\sum_{i=\lambda}^{t-\nu} \theta_{i}\left(\begin{array}{c}
i \\
\lambda
\end{array}\right)\left(\begin{array}{c}
t-i \\
\nu
\end{array}\right) F_{i-\lambda, t-\nu-i}+F_{t-(\lambda+\nu)-1}\right\}+k
$$

where $F_{t-(\lambda+\nu)-1}$ is a functional with a representation of order at most $t-(\lambda+\nu)$ -1 and $k \in[\mathscr{K}]$.

The proof of this corollary follows at once from (20). If we put $\lambda+\nu$ $=t-k$ in (22) we get

CoRollary 3.10. Let $F$ be an invariant functional on $X$ having a representation of order $t>k$ given by (21). Then

$$
0=\sum_{j=0}^{k} \theta_{\lambda+j}\left(\begin{array}{c}
\lambda+j \\
\lambda
\end{array}\right)\left(\begin{array}{c}
t-\lambda-j \\
t-\lambda-k
\end{array}\right) F_{j, k-j} \quad(\text { on } X)
$$

for all $\lambda, 0 \leqq \lambda \leqq t-k$.

Definition 3.11. $\mathscr{T}$ is the space of all functionals on $X$ of the form

$$
\sum_{i+j \leqq t} a_{i, j} F_{i, j} \quad\left(a_{i, j} \in C\right),
$$

which are also invariant on $X$.

Note that the functionals in Theorem 2.5 are contained in $\mathscr{T}$. As a first application of Corollary 3.10 we shall prove the following theorem. 
Theorem 3.12. Let $k=\min \left\{i+j: F_{i, j} \equiv 0\right.$ on $\left.X\right\}$ and let

$$
\mathscr{K}=\left\{F_{i, k-i} \neq 0 \text { on } X\right\} \text {. }
$$

If $\mathscr{K}$ contains $k+1$ linearly independent elements, then $\mathscr{T}=[\mathscr{K}]$.

Proof. We shall show that any invariant functional of the form (21) with $t>k$, must be in [ $\mathscr{K}$ ]. To do this it suffices to show $\theta_{i}=0,0 \leqq i \leqq t$. But from (22) and the hypothesis, we have $\theta_{\lambda}=\theta_{\lambda+1}=\cdots=\theta_{\lambda+k}=0,0 \leqq \lambda \leqq t$ $-k$. Hence $\theta_{0}=\theta_{1}=\cdots=\theta_{t}=0$.

Thus in the case where $\mathscr{K}$ contains $k+1$ linearly independent elements, we see $\operatorname{dim} \mathscr{T}=k+1$. In the next section we shall show that it is always true that $\operatorname{dim} \mathscr{T} \leqq k+1$.

4. The finite-dimensionality of $\mathscr{T}$.

TheOREM 4.1. Let $X$ be an invariant subspace of $L^{\#}\left(R^{2}\right)$ and let $\mathscr{T}$ $=\left\{F=\sum_{i+j \leqq t} a_{i, j} F_{i, j}: F\right.$ is invariant on $\left.X\right\}$. Then $\operatorname{dim} \mathscr{T} \leqq k+1$, where $=\min \left\{i+j: F_{i, j} \not \equiv 0\right.$ on $\left.X\right\}$.

Before giving the proof we shall give a few examples in order to show some of the things that are possible.

A. Let $X_{1}=\left\{f \in L^{\#}\left(R^{2}\right): F_{0,0}(f)=0\right\}$. Then we have seen in $\$ 1$ that $F_{0,1}$ and $F_{1,0}$ are linearly independent on $X$; hence according to Theorem $3.12, \mathscr{T}=\left[F_{0,1}, F_{1,0}\right]$ and $\operatorname{dim} \mathscr{T}=2=k+1$.

B. Let $X_{2}=\left\{f \in X_{1}: F_{0,1}(f)=F_{1,0}(f)\right\}$. The functional $G=F_{2,0}-2 F_{1,1}$ $+F_{0,2}$ is invariant on $X$. The functions $g(x, y)=x \sin x \sin y+y \sin y \sin x$, $|x|,|y| \leqq \pi, g(x, y)=0$ elsewhere, $h(x, y)=\sin x \sin y,|x|,|y| \leqq \pi, h(x, y)$ $=0$ elsewhere, are in $X_{2}$ and show that $F_{1,0}$ and $G$ are linearly independent in $X_{2}$. It follows from the theorem that $\mathscr{T}=\left[F_{1,0}, G\right]$ and $\operatorname{dim} \mathscr{T}=2=k+1$.

C. Let $\alpha \neq 0$ and let $X_{3}=\left\{f \in X_{2}: G(f)=\alpha F_{1,0}(f)\right\}$. Then the functional $H=F_{3,0}-3 F_{2,1}+3 F_{1,2}-F_{0,3}+r F_{2,0}+s F_{1,1}+t F_{0,2}$ is invariant on $X$ if $r, s$ and $t$ satisfy $2 r+s=\alpha, 2 t+s=-\alpha$.

D. Let $X=\left\{f \in L^{\#}\left(R^{2}\right): F_{0, q}(f)=0\right.$ for all $\left.q \geqq 0\right\}$. Here $k=1$ and it is easy to see using Corollary 3.10 , that $\operatorname{dim} \mathscr{T}=1<k+1$.

Proof of Theorem 4.1. The proof of the theorem is rather long and we have to break it up into two cases: the case where $\mathscr{K}$ is an independent set and the case where $\mathscr{K}$ is a dependent set.

Thus, let us first suppose that $\mathscr{K}$ is an independent set. Let

$$
m=\min \left\{i: F_{i, k-i} \in \mathscr{K}\right\}, \quad n=\min \left\{k-i: F_{i, k-i} \in \mathscr{K}\right\}
$$

and let

$$
\mathscr{K}=\left\{F_{m_{1}, k-m_{1}}, F_{m_{2}, k-m_{2}}, \cdots, F_{m_{s}, k-m_{s}}\right\}
$$

where $m=m_{1}<m_{2}<\cdots<m_{s}=k-n$. Let $t>k$ and let $F$ be an invariant functional on $X$ having a representation of order $t$; i.e., $F$ is given by 
(21) where not all $\theta_{i}=0,0 \leqq i \leqq t$. From Corollary 3.10, we see that (23) holds, for all $\lambda, 0 \leqq \lambda \leqq t-k$. Now if $F_{j, k-j} \notin \mathscr{K}$, then $F_{j, k-j}=0$. Therefore, we get

$$
0=\sum_{i=1}^{s} \theta_{\lambda+m_{i}}\left(\begin{array}{c}
\lambda+m_{i} \\
\lambda
\end{array}\right)\left(\begin{array}{c}
t-\lambda-m_{i} \\
t-\lambda-k
\end{array}\right) F_{m_{i}, k-m_{i}} \quad(0 \leqq \lambda \leqq t-k),
$$

and by the independence of $\mathscr{K}$, we have

$$
\theta_{\lambda+m_{i}}=0, \quad 1 \leqq i \leqq s, 0 \leqq \lambda \leqq t-k .
$$

We let $J=\left\{j \in I: m_{j+1}-1 \leqq m_{j}+2,1 \leqq j \leqq s-1\right\}$, and we have the following lemma.

LEMma 1. We can write $F$ as

$$
F=\sum_{i=0}^{m-1} \theta_{i} F_{i, t-i}+\sum_{j \in J} \sum_{i=m_{j}+2}^{m_{j+1}-1} \theta_{i} F_{i, t-i}+\sum_{i=t-n+1}^{t} \theta_{i} F_{i, t-i}+F_{t-1}
$$

Proof. We have

$$
F=\sum_{i=0}^{m-1} \theta_{i} F_{i, t-i}+\sum_{j=1}^{s-1} \sum_{i=m_{j}}^{m_{j+1}-1} \theta_{i} F_{i, t-i}+\sum_{i=m_{g}}^{t} \theta_{i} F_{i, t-i}+F_{t-1} .
$$

Since $t-k \geqq 1$, we have $\theta_{m_{j}}=\theta_{m_{j}+1}=0$ for $1 \leqq j \leqq s-1$; so for $1 \leqq j \leqq s$

$$
\sum_{i=m_{j}}^{m_{j+1}-1} \theta_{i} F_{i, t-i}=\sum_{i=m_{j+2}}^{m_{j+1}-1} \theta_{i} F_{i, t-i}
$$

and hence in (27) we may omit all $j$ for which $m_{j}+2>m_{j+1}-1$ so that

$$
\sum_{j=1}^{s-1} \sum_{i=m_{j}}^{m_{j+1}-1} \theta_{i} F_{i, t-i}=\sum_{j \in J} \sum_{i=m_{j+2}}^{m_{j+1}-1} \theta_{i} F_{i, t-i}
$$

Also if we let $i=s$ in (25) and note that $m_{s}-k=-n$, we get

$$
\sum_{i=m_{8}}^{t} \theta_{i} F_{i, t-i}=\sum_{i=t-n+1}^{t} \theta_{i} F_{i, t-1}
$$

This equation along with (27) and (28) yield (26).

It follows that the number of nonzero $\theta_{i}$ 's appearing in (26) is bounded above independently of $t$. We state this more precisely as

LEMMA 2. If $F$ is an invariant functional having a representation of order $t>k$, then the number of nonzero coefficients in the principal part of $F$ is at most $k+1-s$ where $s=\operatorname{dim} \mathscr{K}$.

The next step in the proof is to show that if we have an invariant func- 
tional $F$ having a representation of order $t>k$ and $r$ is any integer such that $t>r>k$, then we can find an invariant functional having a representation of order $r$ whose principal part is closely related to the principal part of $F$.

Lemma 3. Let $F$ be an invariant functional having a representation of order $t>k$ (which we may assume, by Lemma 1 , is of the form (26)). Let $r$ be any integer with $t>r>k$.

(A) If $\theta_{i_{0}} \neq 0$ for some $i_{0}, 0 \leqq i_{0} \leqq t-n$, then there is a functional $\Phi \in[\mathscr{K}]$ of the form

$$
\begin{aligned}
\Phi=\sum_{i=0}^{m-1}\left(\begin{array}{c}
t-i \\
r-i
\end{array}\right) \theta_{i} F_{i, r-i} & +\sum_{j \in J} \sum_{i=m_{j}+2}^{m_{j+1}-1}\left(\begin{array}{c}
t-i \\
r-i
\end{array}\right) \theta_{i} F_{i, r-i} \\
& +\sum_{i=r-n+1}^{r}\left(\begin{array}{c}
t-i \\
r-i
\end{array}\right) \theta_{i} F_{i, r-i}+\Phi_{r-1} .
\end{aligned}
$$

(B) If $\theta_{i}=0$ for all $i$ with $0 \leqq i \leqq t-n$, then there is a functional $\Psi \in[\mathscr{K}]$ of the form

$$
\Psi=\sum_{i=r-n+1}^{r}\left(\begin{array}{c}
i+t-\eta \\
t-r
\end{array}\right) \theta_{i+t-r} F_{i, r-i}+\Psi_{r-1} .
$$

Proof. We shall first prove (A). In the invariance equation for $F$ we look at the coefficient of $v^{t-r}$. By Corollary 3.9, it is

$$
0=\left\{\sum_{i=0}^{r}\left(\begin{array}{c}
t-i \\
r-i
\end{array}\right) \theta_{i} F_{i, r-i}+\Phi_{r-1}\right\}+k_{0}
$$

where $k_{0} \in[\mathscr{K}]$. We put $\Phi=\{\}$; then $\Phi \in[\mathscr{K}]$ and $\Phi$ has a representation of order $r$. Applying Lemma 1 gives us the desired form for $\Phi$. The proof of (B) is similar; we use Corollary 3.9 to find the coefficient of $u^{t-r}$ in the invariance equation for $F$.

Let $t$ and $r$ be integers, $t \geqq r>k$ and suppose that $F$ and $G$ are invariant functionals having representations of orders $t$ and $r$, respectively. Then we have

$$
\begin{gathered}
F=\sum_{i=0}^{m-1} \theta_{i} F_{i, t-i}+\sum_{j \in J} \sum_{i=m_{j+2}}^{m_{j+1}-1} \theta_{i} F_{i, t-i}+\sum_{i=t-n+1}^{t} \theta_{i} F_{i, t-i}+F_{t-1}, \\
G=\sum_{i=0}^{m-1} \phi_{i} F_{i, r-i}+\sum_{j \in J} \sum_{i=m_{j+2}}^{m_{j+1}-1} \phi_{i} F_{i, r-i}+\sum_{i=r-n+1}^{r} \phi_{i} F_{i, r-i}+G_{r-1},
\end{gathered}
$$

and we see that there is a one-one correspondence between the indices appearing in the principal parts of $F$ and $G$ given by 


$$
\begin{gathered}
i \leftrightarrow i \quad \text { if } 0 \leqq i \leqq m-1 \quad \text { or } \quad m_{j}+2 \leqq i \leqq m_{j+1}-1, \quad j \in J, \\
t-n+x \leftrightarrow r-n+x \quad \text { if } 1 \leqq x \leqq n .
\end{gathered}
$$

Then using Lemma 3, we see that if $F$ is an invariant functional having a representation of order $t>k$ of the form (26) where $\theta_{i_{0}} \neq 0$, and if $t>r>k$ then there is an invariant functional $G=\sum_{i=0}^{r} \phi_{i} F_{i, r-i}+G_{r-1}$ having a representation of order $r$ where $\phi_{i_{0}}=0$. We use this fact to prove

LEMma 4. Let $t$ and $r$ be integers with $t \geqq r>k$ and let $F$ and $G$ be invariant functionals having representations of orders $t$ and $r$ given by (31) and (32), respectively. If $i_{0}$ is chosen so that $\theta_{i_{0}} \neq 0, \theta_{i}=0$ if $i<i_{0}$ then there exists an invariant functional $\bar{G}$ of the form

$$
\bar{G}=\sum_{i=0}^{m-1} \bar{\phi}_{i} F_{i, n-i}+\sum_{j \in J} \sum_{i=m j+2}^{m_{j+1}-1} \bar{\phi}_{i} F_{i, r-i}+\sum_{i=r-n+1}^{r} \bar{\phi}_{i} F_{i, r-i}+G_{r-1},
$$

where $\bar{\phi}_{i}=\phi_{i}$ if $i<i_{0}, \bar{\phi}_{i_{0}}=0$ and $[\mathscr{K}, \bar{G}, F]=[\mathscr{K}, G, F]$.

Proof. If $t=r$ we put $\bar{G}=G-\left(\phi_{i_{0}} / \theta_{i_{0}}\right) F$. If $t>r$ we have two cases to consider, namely, $0 \leqq i_{0} \leqq t-n$ and $t-n<i_{0} \leqq t$. If $0 \leqq i_{0} \leqq t-n$, we apply Lemma $3 \mathrm{~A}$ to obtain

$$
\Phi \in[\mathscr{K}] \text { with }\left[\theta_{i_{0}}\left(\begin{array}{c}
t-i_{0} \\
r-i_{0}
\end{array}\right)\right]^{-1} \neq 0 .
$$

Then we put

$$
\bar{G}=G-\phi_{i_{0}}\left[\theta_{i_{0}}\left(\begin{array}{c}
t-i_{0} \\
r-i_{0}
\end{array}\right)\right]^{-1} \Phi
$$

and $\bar{G}$ has the required properties. If $t-n<i_{0} \leqq t$, we use Lemma $3 \mathrm{~B}$ and a similar argument gives us the desired result.

We shall now select a set of invariant functionals which we shall show forms a basis for $\mathscr{T}$ having at most $k+1$ elements.

Let $z$ be the maximal number of nonzero coefficients appearing in the principal part of any invariant functional having a representation of order $t>k$. From Lemma $2, z \leqq k+1-s$ where $s=\operatorname{dim} \mathscr{K}$. Let

$I_{0}=\{n \in I: \exists$ invariant $F$ with a representation of order $n$ and $F \notin[\mathscr{K}]\}$. If $I_{0}$ is void then $\mathscr{T} \subset[\mathscr{K}]$ and we are done. If $I_{0}$ is nonvoid, we let $p_{0}$ $=\min I_{0}$ and let $H_{0}$ be invariant on $X, H_{0}$ having a representation of order $p_{0}$ and $H_{0} \notin[\mathscr{K}]$. Inductively, choose $H_{\alpha} \in \mathscr{T}$ so that

$$
H_{\alpha} \notin\left[\mathscr{K}, H_{1}, \cdots, H_{\alpha-1}\right]
$$

and so that $H_{\alpha}$ has a representation of minimum order, this order being $p_{\alpha}$. 
If $\operatorname{dim} \mathscr{T}<k+1$, we are done; otherwise $H_{\alpha}$ and $p_{\alpha}$ are defined for $0 \leqq \alpha$ $\leqq z-1$. From Lemma 1 , for $0 \leqq \alpha \leqq z-1$, we have

$$
H_{\alpha}=\sum_{i=0}^{m-1} a_{i}^{\alpha} F_{i, p_{\alpha}-i}+\sum_{j \in J} \sum_{i=m_{j}+2}^{m_{j+1}-1} a_{i}^{\alpha} F_{i, p_{\alpha}-i}+\sum_{i=p_{\alpha}-n+1}^{p_{\alpha}} a_{i}^{\alpha} F_{i, p_{\alpha}-i}+H_{p_{\alpha}-1}
$$

Note that $k<p_{0} \leqq p_{1} \leqq \cdots \leqq p_{z-1}$. Now suppose $H_{z}$ is invariant on $X$ and $H_{z}$ has a representation of order $p_{z}$. We shall show that the assumption $H_{z} \notin\left[\mathscr{K}, H_{0}, \cdots, H_{z-1}\right]$ leads to a contradiction. If $H_{z} \notin\left[\mathscr{K}_{,} H_{0}, \cdots, H_{z-1}\right]$, then $p_{z} \geqq p_{z-1}$.

Let $\left\{i \in I: m_{j}+2 \leqq i \leqq m_{j+1}-1, j \in J\right\}=\left\{i_{1}<i_{2}<\cdots<i_{p}\right\}$, and consider the array of $z$ columns and $z+1$ rows:

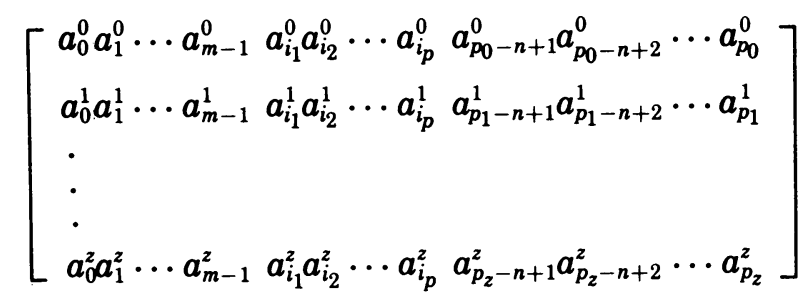

made up of the coefficients of the principal parts of the functionals $H_{\alpha}$, $0 \leqq \alpha \leqq z$. By changing the notation, we give the array the simpler form

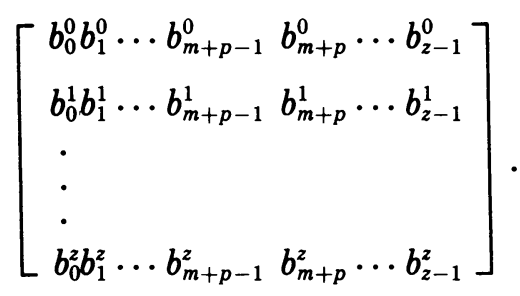

We shall refer to this array as a representation of the functionals $H_{\alpha}$, $0 \leqq \alpha \leqq z$.

Lemma 5. There exist invariant functionals $A_{0}, A_{1}, \cdots, A_{z}$ having a representation $\left[c_{i}^{j}\right], i=0,1, \cdots, z-1 ; j=0,1, \cdots, z$, and which satisfy

(i) $c_{i}^{0}=0, \cdots, 0 \leqq i \leqq z-1$,

(ii) $\left[\mathscr{K}, A_{0}, A_{1}, \cdots, A_{\beta}\right]=\left[\mathscr{K}, H_{0}, H_{1}, \cdots, H_{\beta}\right], 0 \leqq \beta \leqq z$.

Proof. By induction. Since $H_{z}$ has a representation of order $p_{z}$, not all $b_{i}^{z}=0,0 \leqq i \leqq z-1$ in $\left(^{*}\right)$. We define the integer $\epsilon_{0}$ by $b_{\epsilon_{0}}^{z} \neq 0, b_{\epsilon}^{z}=0$ if $\epsilon<\epsilon_{0}$ and we put $B_{z}^{0}=H_{z}, c_{i}^{z}=b_{i}^{z}, 0 \leqq i \leqq z-1$. If $b_{c_{0}}^{r} \neq 0$ for some $r, 0 \leqq r$ $<z$, then we apply Lemma 4 to the functionals $B_{z}^{0}$ and $H_{r}$ to obtain $\bar{H}_{r}$ having coefficients $\bar{b}_{0}^{r}, \bar{b}_{1}^{r} \ldots, \bar{b}_{z-1}^{r}$ on level $p_{r}$ with $\bar{b}_{\epsilon_{0}}^{r}=0$ and $\left[\mathscr{K}, \bar{H}_{r}, B_{z}^{0}\right]$ $=\left[\mathscr{K}, H_{r}, B_{z}^{0}\right]$. Now if $\overline{b_{i}^{r}}=0$ for all $0 \leqq i \leqq z-1$ and $r=0$, then we are done (put $A_{i}=H_{i}, 0 \leqq i \leqq z-1$ and $A_{z}=B_{z}^{0}$ ); if $r>0$, then from the construction in Lemma 4 we obtain a contradiction. Therefore we may 
assume that not all $\bar{b}_{i}^{r}=0,0 \leqq i \leqq z-1$, and we put $B_{r}^{0}=\bar{H}_{r}$. Hence by this method we may assume that we have functionals $B_{i}^{0}, 0 \leqq i \leqq z$, where $B_{i}^{0}$ has a representation of order $p_{i}$ and where the principal part of $B_{i}^{0}, 0 \leqq i$ $\leqq z-1$, with respect to this representation has coefficients $b_{0}^{i}, b_{1}^{i}, \cdots, b_{z-1}^{i}$ and which satisfy

(i) $c_{\epsilon_{0}}^{z} \neq 0, c_{\epsilon}^{z}=0$ if $\epsilon<\epsilon_{0}$,

(ii) $b_{\epsilon_{0}}^{r}=0$ if $0 \leqq r \leqq z-1$,

(iii) $)_{0}\left[\mathscr{K}, B_{0}^{0}, B_{1}^{0}, \cdots, B_{\beta}^{0}\right]=\left[\mathscr{K}, H_{0}, H_{1}, \cdots, H_{\beta}\right], 0 \leqq \beta \leqq z$.

Now suppose that we have invariant functionals $B_{0}^{q}, B_{1}^{q}, \cdots, B_{z}^{q}(q \leqq z-2)$, having representations of orders $p_{0}, p_{1}, \cdots, p_{z}$, respectively, with the coefficients of the principal part of $B_{i}^{q}$ with respect to this representation being

$$
\begin{array}{ll}
b_{0}^{i}, b_{1}^{i}, \cdots, b_{z-1}^{i}, & 0 \leqq i<z-q, \\
c_{0}^{i}, c_{1}^{i}, \cdots, c_{z-1}^{i}, & z-q \leqq i \leqq z,
\end{array}
$$

and suppose we have integers $\epsilon_{0}, \epsilon_{1}, \cdots, \epsilon_{q}$ such that the following conditions hold:

(i) $c_{q} c_{\epsilon_{\alpha}}^{2-\alpha} \neq 0, c_{\epsilon}^{2-\alpha}=0 \quad$ if $\epsilon<\epsilon_{\alpha}(0 \leqq \alpha \leqq q)$,

(ii) $)_{q} c_{\epsilon_{\alpha}}^{r}=0 \quad$ if $z-q \leqq r<z-\alpha$,

(iii) $q \quad b_{\epsilon_{\alpha}}^{r}=0 \quad$ if $0 \leqq r<z-q$,

$$
\left[\mathscr{K}, B_{0}^{q}, B_{1}^{q}, \cdots, B_{\beta}^{q}\right]=\left[\mathscr{K}, H_{0}, H_{1}, \cdots, H_{\beta}\right], \quad 0 \leqq \beta \leqq z .
$$

We shall show how to obtain functionals $B_{0}^{q+1}, B_{1}^{q+1}, \ldots, B_{z}^{q+1}$, and $\epsilon_{q+1}$ satisfying (i) $q+1,(\text { ii) })_{q+1}$ and (iii) $)_{q+1}$.

Since $B_{z-q-1}^{q}$ has a representation of order $p_{z-q-1}$, not all $b_{i}^{z-q-1}=0,0 \leqq i$ $\leqq z-1$. Let $\epsilon_{q+1}$ be defined by $b_{\epsilon_{q+1}}^{z-q-1} \neq 0, b_{\epsilon}^{z-q-1}=0$ if $\epsilon<\epsilon_{q+1}$, and put $B_{i}^{q+1}$ $=B_{i}^{q}, \quad z-q-1 \leqq i \leqq z, c_{i}^{z-q-1}=b_{i}^{z-q-1}, 0 \leqq i \leqq z-1$. Then (i) $)_{q+1}$ and (iii) $_{q+1}$ are satisfied. We shall show how to satisfy (ii) $)_{q+1}$. If $b_{\varepsilon q+1}^{l} \neq 0$ for some $0 \leqq l<z-q-1$, then we apply Lemma 4 to the functionals $B_{l}^{q}, B_{z-q-1}^{q+1}$ to obtain a functional $\bar{B}^{q}$ having coefficients $\bar{b}_{0}^{l}, \bar{b}_{1}^{l}, \ldots, \bar{b}_{z-1}^{l}$ on level $p_{l}$ with $\bar{b}_{\epsilon_{q+1}^{l}}^{l}=0$, and $\left[\mathscr{K}, \bar{B}_{l}^{q}, B_{\underline{z}-q-1}^{q+1}\right]=\left[\mathscr{K}, B_{l}^{q} B_{z-q-1}^{q+1}\right]$. Moreover, as before we may assume that not all $\bar{b}_{i}^{l}=0,0 \leqq i \leqq z-1$. Now if $\bar{b}_{\epsilon_{i_{0}}^{l}} \neq 0$ with $i_{0}<q+1$ (i.e., if by our application of Lemma 4 we have messed up a place that was fixed up previously), then it must be that $\epsilon_{i_{0}}>\epsilon_{q+1}$. We apply Lemma 4 again to the functionals $\bar{B}_{l}^{q}$ and $B_{z-i_{0}}^{q+1}$ to obtain a functional $B_{l}^{\prime q}$ having coefficients $\bar{b}_{i}^{l}, 0 \leqq i \leqq z-1$ on level $p_{l}$ where $\bar{b}_{\epsilon_{q+1}^{l}}=\bar{b}_{\epsilon_{i_{0}}^{l}}=0$ and $\left[\mathscr{K}_{1}, \bar{B}_{l}^{\prime q}, B_{z-i_{0}}^{q+1}\right]=\left[\mathscr{K}_{1}, \bar{B}_{l}^{q}, B_{z-i_{0}}^{q+1}\right]$. Moreover, we may again assume that not all ${\overline{b_{i}^{\prime}}}^{\prime}=0,0 \leqq i \leqq z-1$. Here again we can only mess up a $\overline{b_{i}^{\prime}}$ with $\epsilon_{i}$ $>\epsilon_{i_{0}}$ so that by repeated application of this method we finally satisfy (ii) $)_{q+1}$ and since our applications of Lemma 4 do not effect (i) $)_{q+1}$ or (iii) $)_{q+1}$, we see that by induction (i) $)_{z-1},(\text { ii) })_{z-1}$ and (iii) $)_{-1}$ hold. We put $c_{i}^{0}=b_{i}^{0}$, 
$0 \leqq i \leqq z-1$, and $A_{i}=B_{i}^{2-1}, 0 \leqq i \leqq z$ and we see that the proof of the lemma is complete.

We are now ready to obtain the desired contradiction. From (i) in Lemma $5, A_{0}$ has a representation of order less than $p_{0}$ so by definition of $p_{0}, A_{0}$ $\in[\mathscr{K}]$. From (iii) of Lemma 5 , with $\beta=0$, we have $\left[\mathscr{K}, H_{0}\right]=\left[\mathscr{K}, A_{0}\right]$ $=[\mathscr{K}]$ so that $H_{0} \in[\mathscr{K}]$, a contradiction. Thus the theorem is proved in the case where $\mathscr{K}$ is an independent set.

We shall now consider the case where $\mathscr{K}$ is a dependent set. The main idea is to obtain for any $F \in \mathscr{J}$ of order $t>k$, a representation analogous to (24). Once this has been done, the proof follows just as in the previous case.

Thus, let $\mathscr{K}$ be a dependent set and let $m$ and $n$ be defined as before. We choose a maximal independent subset

$$
\mathscr{K}_{I}=\left\{F_{m_{1}, k-m_{1}}, F_{m_{2}, k-m_{2}}, \cdots, F_{m_{s}, k-m_{s}}\right\} \quad\left(m \leqq m_{1}<m_{2}<\cdots<m_{s}=k-n\right)
$$

of $\mathscr{K}$ having largest possible second indices. We shall suppose that $m<m_{1}$; the case where $m=m_{1}$ is treated similarly. Then we can write $\mathscr{K}$ as

$$
\begin{aligned}
\mathscr{K}=\left\{F_{m_{1}^{1}, k-m_{1}}^{1}, \mathrm{~F}_{m_{2}^{1}, k-m_{2}^{1}}^{1}, \cdots, F_{m_{r_{1}}^{1}, k-m_{r_{1}}^{1}}, F_{m_{1}, k-m_{1}}, \cdots,\right. \\
\left.F_{m_{1}^{s}, k-m_{1}^{s}}, F_{m_{2}^{s}, k-m_{2}^{s}}^{s}, \cdots, F_{m_{r_{s}}^{s}, k-m_{r_{s}}^{s}}, F_{m_{s}, k-m_{s}}\right\},
\end{aligned}
$$

where $m=m_{1}^{1}<m_{2}^{1}<\cdots<m_{r_{1}}^{1}<m_{1}<\cdots<m_{1}^{s}<m_{2}^{s}<\cdots<m_{r_{s}}^{s}<m_{s}=k$ $-n$. We have chosen $\mathscr{K}_{I}$ so that the following relations hold for some $\alpha_{i}^{(h, j)} \in C:$

$$
F_{m_{j, k-m_{j}}^{h}}=\sum_{i=h}^{s} \alpha_{i}^{(h, j)} F_{m_{i}, k-m_{i}} \quad\left(1 \leqq j \leqq r_{h}, 1 \leqq h \leqq s\right) .
$$

Now suppose $t>k$ and

$$
F=\sum_{i=0}^{t} a_{i} F_{i, t-i}+F_{t-1}
$$

is an invariant functional on $X$ of order $t>k$. Our aim is to prove a result like Lemma 1. The first step in this direction is the following lemma.

Lemma 6. Let $i$ be an integer such that $1 \leqq i \leqq s$. Then for $0 \leqq \lambda \leqq t-k$, we have

$$
a_{\lambda+m_{i}}=\sum_{j=m_{1}^{1}}^{m_{i}-1}\left(\begin{array}{c}
t-j \\
\lambda+m_{i}-j
\end{array}\right) c_{j}^{(\lambda, i)} a_{j}
$$

where $c_{j}^{(\lambda, l)}$ is independent of $t$.

Proof. From Corollary 3.10, 


$$
0=\sum_{j=0}^{k} a_{j+\lambda}\left(\begin{array}{c}
\lambda+j \\
\lambda
\end{array}\right)\left(\begin{array}{c}
t-\lambda-j \\
k-j
\end{array}\right) F_{j, k-j}, \quad 0 \leqq \lambda \leqq t-k,
$$

where, as before the sum is taken over those $j$ for which $F_{j, k-j} \in \mathscr{K}$. If we use (35) in (37) and use the independence of $\mathscr{K}_{I}$, we have, for $1 \leqq i \leqq s$,

$$
0=\sum_{h=m_{1}^{1}}^{m_{i}-1} a_{\lambda+h}\left(\begin{array}{c}
\lambda+h \\
\lambda
\end{array}\right)\left(\begin{array}{c}
t-\lambda-h \\
k-h
\end{array}\right) \beta_{h}^{i}+a_{\lambda+m_{i}}\left(\begin{array}{c}
\lambda+m_{i} \\
\lambda
\end{array}\right)\left(\begin{array}{c}
t-\lambda-m_{i} \\
k-m_{i}
\end{array}\right)
$$

for $0 \leqq \lambda \leqq t-k$, where we define

$$
\begin{aligned}
\beta_{h}^{i} & =\alpha_{i}^{(p, q)}, & & \text { if } h=m_{q}^{p}, 1 \leqq q \leqq r_{p}, 1 \leqq p \leqq i, \\
& =0, & & \text { otherwise. }
\end{aligned}
$$

We shall show that (38) implies (36). We define

$$
z_{h}^{\lambda}=-\left(\begin{array}{c}
t-\lambda-h \\
m_{i_{i}}-h
\end{array}\right)\left(\begin{array}{c}
\lambda+h \\
\lambda
\end{array}\right)\left(\begin{array}{c}
k-h \\
m_{j}-h
\end{array}\right)^{-1} \frac{\left(m_{i}\right) ! \lambda !}{\left(\lambda+m_{i}\right) !}
$$

then we can write (38) as

$$
a_{\lambda+m_{i}}=\sum_{h=m_{1}^{1}}^{m_{i}-1} a_{\lambda+h} z_{h}^{\lambda} \beta_{h}^{i}, \quad 0 \leqq \lambda \leqq t-k, 1 \leqq i \leqq s .
$$

We perform an induction on $\lambda$. For $\lambda=0$,

$$
a_{m_{i}}=\sum_{h=m_{1}^{1}}^{m_{i}-1} a_{h} z_{h}^{0} \beta_{h}^{i}=\sum_{h=m_{1}^{1}}^{m_{i}-1} a_{h}\left(\begin{array}{c}
t-h \\
m_{i}-h
\end{array}\right)\left[-\left(\begin{array}{c}
k-h \\
m_{i}-h
\end{array}\right)^{-1} \beta_{h}^{i}\right],
$$

and if we put

$$
c_{h}^{(0, i)}=-\left(\begin{array}{c}
k-h \\
m_{i}-h
\end{array}\right)^{-1} \beta_{h}^{i},
$$

we see that (36) holds for $\lambda=0$. Now suppose (36) holds for all integers $\lambda, \lambda \leqq \nu-1<t-k$; we shall show (36) holds for $\lambda=\nu$. From (40) we have

$$
a_{\nu+m_{i}}=\sum_{h=m_{1}^{1}}^{m_{i}-1} a_{\nu+h} z_{h}^{\lambda} \beta_{h}^{i}
$$

Also, by our induction hypothesis,

$$
a_{h}=\sum_{j=m_{1}^{1}}^{m_{i}-1}\left(\begin{array}{l}
t-j \\
h-j
\end{array}\right) c_{j}^{\left(h-m_{i}, i\right)} a_{j}, \quad m_{i} \leqq h \leqq m_{i}+\nu-1 .
$$

We consider the case where $m_{i} \leqq m_{1}^{1}+\nu$ and leave for the reader the case 
where $m_{i}-1 \geqq m_{1}^{1}+\nu$. In the former case, (42) holds for all $h$ with $m_{1}^{1}+\nu$ $\leqq h \leqq m_{i}+\nu-1$ so

$$
\begin{aligned}
a_{\nu+m_{i}} & =\sum_{h=\nu+m_{1}^{1}}^{m_{i}+\nu-1} a_{h} z_{h-\nu} \beta_{h-\nu}^{i} \\
& =\sum_{j=m_{1}^{1}}^{m_{i}-1} a_{j} \sum_{h=\nu+m_{1}^{1}}^{m_{i}+\nu-1}\left(\begin{array}{c}
t-j \\
h-j
\end{array}\right) z_{h-\nu}^{\nu} c_{j}^{\left(h-m_{i}, i\right)} \beta_{h-\nu}^{i}
\end{aligned}
$$

Now

$$
\left(\begin{array}{c}
t-j \\
h-j
\end{array}\right) z_{h-\nu}^{\nu}=\left(\begin{array}{c}
t-j \\
\nu+m_{i}-j
\end{array}\right) d(\nu, h, j),
$$

where $d(\nu, h, j)$ is independent of $t$. Then putting this back into (43) gives (36) for $\lambda=\nu$ if we let

$$
c_{j}^{(\nu, i)}=\sum_{h=\nu+m_{1}^{1}}^{m_{i}+\nu-1} d(\nu, h, j) c_{j}^{\left(h-m_{i}, i\right)} \beta_{h-j}^{i}
$$

Therefore the proof of the lemma is complete by induction.

Now we still must refine (36) further. To this end, let

$$
H=\left\{j \in I: m_{j}-1 \geqq m_{j-1}+1,1 \leqq j \leqq s\right\} .
$$

Note that if $j \notin H$ and $1 \leqq j \leqq s$, then $m_{j-1}+1=m_{j}$. We define $m_{0}+1$ $=m_{1}^{1}(=m)$ and then $1 \in H$.

LEMma 7. Let $i$ be an integer such that $1 \leqq i \leqq s$. Then

$$
a_{\lambda+m_{i}}=\sum_{h \in H ; i \geqq h \geqq 1}\left[\sum_{j=m_{h-1}+1}^{m_{h}-1}\left(\begin{array}{c}
t-j \\
\lambda+m_{i}-j
\end{array}\right) d_{j}^{(\lambda, i)} a_{j}\right] \quad(0 \leqq \lambda \leqq t-k),
$$

where $d_{j}^{(\lambda, i)}$ is independent of $t$.

Proof. By induction on $i$. The case $i=1$ follows at once from (36). Now suppose that (44) holds for all integers $i$ with $i \leqq p-1<s$; we shall show that (26) holds for $i=p$. From (36), we can write

$$
\begin{aligned}
a_{\lambda+m_{p}} & =\sum_{h \in H ; p \geqq h \geqq 1}\left[\sum_{j=m_{h-1}+1}^{m_{h}-1}\left(\begin{array}{c}
t-j \\
\lambda+m_{p}-j
\end{array}\right) c_{j}^{(\lambda, p)} a_{j}\right] \\
& +\sum_{i=2}^{p}\left(\begin{array}{c}
t-m_{i-1} \\
\lambda+m_{p}-m_{i-1}
\end{array}\right) c_{m_{i-1}}^{(\lambda, p)} a_{m_{i-1}} .
\end{aligned}
$$

Now for any $i$ with $2 \leqq i \leqq p$, we use our induction hypothesis with $\lambda=0$ in (45) to obtain 


$$
\begin{aligned}
& a_{\lambda+m_{p}}=\sum_{h \in H ; p \geqq h \geqq 1}\left[\sum_{j=m_{h-1}+1}^{m_{h}-1}\left(\begin{array}{c}
t-j \\
\lambda+m_{p}-j
\end{array}\right) c_{j}^{(\lambda, p)} a_{j}\right] \\
& \quad+\sum_{i=2}^{p}\left[\sum_{h \in H ; i-1 \geqq h \geqq 1} \sum_{j=m_{h-1}+1}^{m_{h}-1}\left(\begin{array}{c}
t-j \\
\lambda+m_{p}-j
\end{array}\right) f_{i-1, j}^{(\lambda, p)} a_{j}\right], \quad 0 \leqq \lambda \leqq t-k,
\end{aligned}
$$

where

$$
f_{i-1}^{(\lambda, p)}=\left(\begin{array}{c}
\lambda+m_{p}-j \\
m_{i-1}-j
\end{array}\right) c_{m_{i-1}}^{(\lambda, p)} d_{j}^{(0, i-1)}
$$

is independent of $t$. Now if $p=2$, then (46) yields (44). Suppose $p>2$. Then we let

$$
B_{i-1}=\sum_{h \in H ; i-1 \geqq h \geqq 1}\left[\sum_{j=m_{h-1}+1}^{m_{h}-1}\left(\begin{array}{c}
t-j \\
\lambda+m_{p}-j
\end{array}\right) f_{i-1, j}^{(\lambda, p)} a_{j}\right], \quad 2 \leqq i \leqq p,
$$

and we can show that

$$
B_{i-1}+B_{i}=\sum_{h \in H ; i \geqq h \geqq 1}\left[\sum_{j=m_{h-1}+1}^{m_{h}-1}\left(\begin{array}{c}
t-j \\
\lambda+m_{p}-j
\end{array}\right) \bar{f}_{i, j}^{(\lambda, p)} a_{j}\right],
$$

where $\bar{f}_{i, j}^{(\lambda, p)}$ is independent of $t$. It follows that we can write (46) as

$$
\begin{aligned}
& a_{\lambda+m_{p}}= \sum_{h \in H ; p \geqq h \geqq 1}\left[\sum_{j=m_{h-1}+1}^{m_{h}-1}\left(\begin{array}{c}
t-j \\
\lambda+m_{p}-j
\end{array}\right) c_{j}^{(\lambda, p)} a_{j}\right] \\
&+\sum_{h \in H ; p-1 \geqq h \geqq 1}\left[\sum_{j=m_{h-1}+1}^{m_{h}-1}\left(\begin{array}{c}
t-j \\
\lambda+m_{p}-j
\end{array}\right) g_{j}^{(\lambda, p)} a_{j}\right], \quad 0 \leqq \lambda \leqq t-k,
\end{aligned}
$$

where $g_{j}^{(\lambda, p)}$ is independent of $t$, and this implies (44) for $i=p$. Therefore the proof of the lemma is complete by induction.

Now we may write $(48)$, for $1 \leqq i \leqq s$, as

$$
a_{\lambda+m_{i}}=\sum_{h \in H} \sum_{j=m_{h-1}+1}^{m_{h}-1}\left(\begin{array}{c}
t-j \\
\lambda+m_{i}-1
\end{array}\right) d_{j}^{(\lambda, i)} a_{j}, \quad 0 \leqq \lambda \leqq t-k
$$

where if $i<h \leqq s, h \in H$, then $d_{j}^{(\lambda, i)}=0$. We shall use (49) in our expression for $F$. We let

$$
\begin{aligned}
& M=\sum_{j=0}^{m-1} a_{j} F_{j, t-j}, \quad N=\sum_{j=t-n+1}^{t} a_{j} F_{j, t-j}, \\
& P=\sum_{j=m_{s}}^{t-n} a_{j} F_{j, t-j}, \quad Q=F-\left(M+N+P+F_{t-1}\right),
\end{aligned}
$$


and first consider $Q$. If $2 \leqq h \leqq s$, and $h \notin H$, then $m_{h-1}+1=m_{h}$ so we can write $Q$ as

$$
Q=\sum_{h \in H} \sum_{j=m_{h-1}+1}^{m_{h}-1} a_{j} F_{j, t-j}+\sum_{i=2}^{s} a_{m_{i-1}} F_{m_{i-1}, t-m_{i-1}} .
$$

If we use (49) with $\lambda=0$ in the second sum we get

$$
Q=\sum_{h \in H} \sum_{j=m_{h-1}+1}^{m_{h}-1} a_{j} F_{j, t-j}+\sum_{i=2}^{s}\left(\begin{array}{c}
t-j \\
m_{i-1}-j
\end{array}\right) d_{j}^{(0, i-1)} F_{m_{i-1}, t-m_{i-1}} .
$$

We next consider $P$. We make the change of variable $\lambda+k-n=j$ and use (49) with $i=s$ to get

$$
\begin{aligned}
P & =\sum_{\lambda=0}^{t-k} a_{\lambda+m_{s}} F_{\lambda+m_{g}, t-\lambda-m_{s}} \\
& =\sum_{\lambda=0}^{t-k}\left[\sum_{h \in H} \sum_{j=m_{h-1+1}}^{m_{h}-1}\left(\begin{array}{c}
t-j \\
\lambda+m_{s}-j
\end{array}\right) d_{j}^{(\lambda, s)} a_{j}\right] F_{\lambda+m_{s}, t-\lambda-m_{g} .}
\end{aligned}
$$

We then get the following expression for $F$ :

$$
\begin{aligned}
& F=\sum_{j=0}^{m-1} a_{j} F_{j, t-j}+\sum_{h \in H} \sum_{j=m_{h-1}+1}^{m_{h}-1} a_{j}\left[F_{j, t-j}+\sum_{i=2}^{s}\left(\begin{array}{c}
t-J \\
m_{i-1}-j
\end{array}\right) d_{j}^{(0, i-1)} F_{m_{i-1}, t-m_{i-1}}\right. \\
& \left.+\sum_{\lambda=0}^{t-k}\left(\begin{array}{c}
t-j \\
\lambda+m_{s}-1
\end{array}\right) d_{j}^{(\lambda, s)} F_{\lambda+m_{g}, t-\lambda-m_{s}}\right]
\end{aligned}
$$

$$
+\sum_{j=t-h+1}^{t} a_{j} F_{j, t-j}+F_{t-1}
$$

Thus we have shown that any invariant functional $F$ having a representation of order $t>k$ can be put in the form (53). This is the analogue of (26) in the case where $\mathscr{K}$ is a dependent set. We see from (53), that the number of nonzero coefficients appearing in the principal part of $F$ is again at most $k+1-s$ where $s$ is the dimension of $\mathscr{K}$. The rest of the proof of the theorem proceeds exactly as in the case where $\mathscr{K}$ is an independent set.

CONJECTURE. If $X$ is an invariant subspace of $L^{\#}\left(R^{N}\right)$ where $N \geqq 1$ and if

$$
\mathscr{T}=\left\{F=\sum_{i_{1}+\cdots+i_{N} \leqq t} a_{i_{1} \cdots i_{N}} F_{i_{1}, \cdots, i_{N}}: F \text { is invariant on } X\right\},
$$

then

$$
\operatorname{dim} \mathscr{T} \leqq\left(\begin{array}{c}
k+N-1 \\
N-1
\end{array}\right)
$$


(Here $F_{i_{1}, \cdots, i_{N}}$ is defined in a manner analogous to (11), and $k$ is defined in a manner analogous to (12).)

\section{REFERENCES}

1.S. Bochner and K. Chandrasekharan, Fourier transforms, Princeton Univ. Press, Princeton, N. J., 1949.

2. M. Jerison and W. Rudin, Translation-invariant functionals, Proc. Amer. Math. Soc. 13 (1962), 417-423.

3. L. Schwartz, Théorie des distributions, Tomes 1, 2, Hermann, Paris, 1950, 1951.

Purdue University,

LAFA YETTE, INDIANA 